\title{
Sharkovskiü's theorem holds for some discontinuous functions
}

by

\author{
Piotr Szuca (Gdańsk)
}

\begin{abstract}
We show that the Sharkovskiur ordering of periods of a continuous real function is also valid for every function with connected $G_{\delta}$ graph. In particular, it is valid for every $\mathrm{DB}_{1}$ function and therefore for every derivative. As a tool we apply an Itinerary Lemma for functions with connected $G_{\delta}$ graph.
\end{abstract}

1. Introduction. Consider the following ordering of the natural numbers:

$$
\begin{gathered}
3 \triangleright 5 \triangleright 7 \triangleright \ldots \triangleright 2 \cdot 3 \triangleright 2 \cdot 5 \triangleright 2 \cdot 7 \triangleright \ldots \triangleright 2^{2} \cdot 3 \triangleright 2^{2} \cdot 5 \triangleright 2^{2} \cdot 7 \triangleright \ldots \\
\ldots \triangleright 2^{4} \triangleright 2^{3} \triangleright 2^{2} \triangleright 2 \triangleright 1 .
\end{gathered}
$$

(First list all odd numbers except 1, followed by 2 times the odds, 4 times the odds, etc. Next list all powers of two in decreasing order.) We reserve the symbols " $\triangleright$ " and " $\triangleleft$ " for that order and call it Sharkovskiน's ordering.

Sharkovski1's theorem says that for every continuous map $f: \mathbb{R} \rightarrow \mathbb{R}$, if $f$ has a point of prime period $P$ then it also has a point of prime period $Q$ for each $Q \triangleleft P$. The remarkable thing about it is a weak assumption (the function is only assumed to be continuous) and strong conclusion. In this paper we will show that the assumption can even be weakened - it is enough to assume the function to be a connected $G_{\delta}$ subset of $\mathbb{R}^{2}$ (see definitions below). This is surprising, since there are examples of functions in this class with some pathological properties. In particular, such a function can be discontinuous on a set of positive Lebesgue measure.

One of the reasons for studying fixed point properties of such functions is the lack of a nice characterization of derivatives. The latter are $\mathrm{DB}_{1}$

2000 Mathematics Subject Classification: Primary 26A15; Secondary 26A18, 26A21, 54C30, 37E99.

Key words and phrases: periodic points, iteration, Itinerary Lemma, Sharkovskiı̌'s ordering, derivatives, connectivity functions, Darboux functions, Baire 1 functions, Borel measurable functions.

This work was partially supported by grant number BW/5100-5-0085-3. 
(a subset of connected $G_{\delta}$ real functions), but not every $\mathrm{DB}_{1}$ function is a derivative. It is easy to see that the composition of derivatives has the Darboux property, but it does not need to be a derivative. Since functions with connected graph have the Darboux property, it seems interesting to find out if the composition of derivatives is connected. The question if the composition of two derivatives has a fixed point is an easier version of this problem. (Every bounded connected function defined on $\mathbb{R}$ has a fixed point, but there are examples of bounded Darboux functions without any.) An affirmative answer to this question was recently given by Csörnyei, O'Neil, and Preiss [2], and independently by Elekes, Keleti, and Prokaj [4] (results from [2] and [4] can be generalized to connected $G_{\delta}$ functions).

Another problem regarding fixed point properties of $\mathrm{DB}_{1}$ functions was posed by Kellum in [7]. He constructed an example of a connectivity function $f: \mathbb{R} \rightarrow \mathbb{R}$ which has a point of prime period 3 but for each $x$, either $f^{3}(x)=$ $x$, or $x$ is not equal to $f^{N}(x)$ for any $N>0$. This shows that Sharkovskiil's theorem cannot be extended to all connectivity functions. Kellum asked whether it can be generalized to the class of Darboux Baire 1 real functions. Our result gives an affirmative answer to this question.

We prove that if $f: \mathbb{R} \rightarrow \mathbb{R}$ is function with connected $G_{\delta}$ graph, $N \in \mathbb{N}$, and $f^{N}$ has a point of prime period $P$ then it also has a point of prime period $Q$ for each $Q \triangleleft P$ (Theorem 3.4). As a tool we apply a fact known in one dimensional combinatorial dynamics of continuous functions as the "Itinerary Lemma" (Corollary 1).

It is not hard to modify some existing examples (e.g. Example 2.3 of [1]) to produce a Darboux $G_{\delta}$ function $g:[0,1] \rightarrow[0,1]$ without fixed points, such that $g^{2}$ has one. Thus, since $2 \triangleright 1$, the main result of this paper (Theorem 3.4) cannot be extended to the class of all Darboux $G_{\delta}$ functions (but see also the "Problems" section).

We would like to thank Kenneth Kellum for drawing our attention to this problem. In particular, Kellum noticed that the existence of a point of period 3 implies the existence of points of period 2 and 1 for every $\mathrm{DB}_{1}$ function.

2. Preliminaries. When considering real intervals, $[a, b]$ or $(a, b)$, we do not assume that $a<b$. For every $A \subset \mathbb{R}^{2}$ we denote by $A^{-1}$ the set $\{\langle y, x\rangle \mid\langle x, y\rangle \in A\}$. By rectangle we understand a rectangle with sides parallel to the coordinate axes.

We identify every function with its graph. We consider the following classes of functions from $\mathbb{R}$ into $\mathbb{R}$ :

D: $f$ is Darboux $(f \in \mathrm{D})$ if $f(I)$ is an interval for every interval $I \subset \mathbb{R}$; equivalently, $f$ has the intermediate value property; 
Conn: $f$ is connected ( $f \in$ Conn) if $f$ is a connected subset of $\mathbb{R}^{2}$; we will also call such a function a connectivity function;

$\mathrm{B}_{1}$ : $f$ is Baire class 1 if $f$ is the pointwise limit of a sequence of continuous functions; this is equivalent to $f^{-1}(G)$ being an $F_{\sigma}$ subset of $\mathbb{R}$ for every open set $G \subset \mathbb{R}$;

$\mathrm{DB}_{1}: f$ is Darboux Baire $1\left(f \in \mathrm{DB}_{1}\right)$ if $f$ is Darboux and Baire 1 ;

$G_{\delta}: f \in G_{\delta}$ if $f$ is a $G_{\delta}$ subset of $\mathbb{R}^{2}$, i.e. $f=\bigcap_{n \in \mathbb{N}} G_{n}$, where all $G_{n} \subset \mathbb{R}^{2}$ are open.

For properties of these and other Darboux-like classes of functions see e.g. the survey [5]. In particular, it is known that Conn $\subset \mathrm{D}$ and $\mathrm{DB}_{1} \subset$ Conn $\cap G_{\delta}$ (both inclusions are proper). It follows that Conn $=\mathrm{D}$ within the class of Baire 1 functions. Moreover, it is easy to show that if $f \in$ Conn then $f\lceil I$ is connected for every interval $I \subset \mathbb{R}$. In what follows we will also use the fact that every Darboux function is bilaterally dense in itself.

We will apply the following property of connectivity functions.

REMARK 2.1. Suppose that $f: \mathbb{R} \rightarrow \mathbb{R}$ is a connectivity function, $A \cup B$ $\subset f, A \cap B=\emptyset$, and $A \cup B$ is dense in $f$. If $\langle a, f(a)\rangle \in A$ and $\langle b, f(b)\rangle \in B$ for some $a, b \in \mathbb{R}$, then there exists $c \in[a, b]$ such that for every open neighbourhood $U$ of $\langle c, f(c)\rangle, U \cap A \neq \emptyset$ and $U \cap B \neq \emptyset$.

Proof. Let $\operatorname{cl}_{f}(X)$ denote the closure of the set $X$ in the space $f$. Define

$$
A_{0}=A \cap([a, b] \times \mathbb{R}), \quad B_{0}=B \cap([a, b] \times \mathbb{R}) .
$$

Clearly $f\left\lceil[a, b]=\operatorname{cl}_{f}\left(A_{0} \cup B_{0}\right)=\operatorname{cl}_{f}\left(A_{0}\right) \cup \operatorname{cl}_{f}\left(B_{0}\right)\right.$. Since $\langle a, f(a)\rangle \in A_{0}$ and $\langle b, f(b)\rangle \in B_{0}$, we have $A_{0} \neq \emptyset \neq B_{0}$. Since $f\lceil[a, b]$ is connected, it follows that $\mathrm{cl}_{f}\left(A_{0}\right) \cap \mathrm{cl}_{f}\left(B_{0}\right) \neq \emptyset$. Therefore as $\langle c, f(c)\rangle$ we can take any point from $\operatorname{cl}_{f}\left(A_{0}\right) \cap \operatorname{cl}_{f}\left(B_{0}\right)$.

Having a fixed number $N$ we will count naturals modulo $N$, i.e. $N+i=i$ if $0 \leq i<N$.

Fix $f: \mathbb{R} \rightarrow \mathbb{R}$ and $N \in \mathbb{N}$. We write $f^{N}$ for $f \circ \ldots \circ f(N$ times $)$. Additionally, $f^{0}$ is the identity map of $\mathbb{R}$. A point $x \in \mathbb{R}$ such that $f^{N}(x)=x$ but $f^{i}(x) \neq x$ for $i=1, \ldots, N-1$ is called a periodic point of $f$ of prime period $N$. The orbit of such an $x$ is $\left\{f^{M}(x)\right\}_{0 \leq M \leq N-1}$.

We will use the following observation due to Ciesielski.

REMARK 2.2. The composition of functions $f, g: \mathbb{R} \rightarrow \mathbb{R}$ has a fixed point if and only if $f \cap g^{-1} \neq \emptyset$.

We say that a closed interval $I_{1} f$-covers a closed interval $I_{2}$ if $f\left(I_{1}\right) \supset I_{2}$; we then write $I_{1} \rightarrow f I_{2}$ (or $I_{1} \rightarrow I_{2}$ if $f$ is clear from context). Note the following property of this relation. 
Remark 2.3. Suppose that $M \in \mathbb{N}$ and $f=g^{M}$ for $g: \mathbb{R} \rightarrow \mathbb{R}$ being a Darboux function. If $I_{1}, I_{2} \subset \mathbb{R}$ are closed intervals such that $I_{1} \rightarrow_{f} I_{2}$, then there exist closed intervals $J_{1}, \ldots, J_{M+1}$ such that $I_{1}=J_{1} \rightarrow g \ldots \rightarrow g$ $J_{M+1}=I_{2}$.

We say that $f: \mathbb{R} \rightarrow \mathbb{R}$ belongs to the class $\mathcal{I}$ (or has the property $\mathcal{I}$ ) if for every family $\left\{I_{k}\right\}_{1 \leq k \leq N}$ of closed intervals such that $I_{1} \rightarrow_{f} \ldots \rightarrow_{f} I_{N} \rightarrow_{f} I_{1}$ there exists an $x \in I_{1}$ such that $f^{N}(x)=x$ and $f^{i}(x) \in I_{i+1}$ for every $i=1, \ldots, N-1$.

It is not hard to observe that every continuous function has the property $\mathcal{I}$ - this fact is known in one-dimensional dynamics as the "Itinerary Lemma". One of our aims is to prove it for every function with connected $G_{\delta}$ graph.

REMARK 2.4. Suppose that $g: \mathbb{R} \rightarrow \mathbb{R}$ is a Darboux function with the property $\mathcal{I}$. Then $g^{M} \in \mathcal{I}$ for every $M \in \mathbb{N}$.

Proof. Let $M, N \in \mathbb{N}, f=g^{M}$ and $\left\{I_{k}\right\}_{1 \leq k \leq N}$ be a family of closed intervals such that $I_{1} \rightarrow_{f} \ldots \rightarrow_{f} I_{N} \rightarrow_{f} I_{1}$.

First, by Remark 2.3 for every $i=1, \ldots, N$ we can find $M+1$ closed intervals $I^{(i-1) \cdot M+1}, I^{(i-1) \cdot M+2}, \ldots, I^{(i-1) \cdot M+M+1}$ such that

$$
I_{i}=I^{(i-1) \cdot M+1} \rightarrow_{g} I^{(i-1) \cdot M+2} \rightarrow g \ldots \rightarrow g I^{(i-1) \cdot M+M+1}=I_{i+1} .
$$

Next, since $g \in \mathcal{I}$, there exists an $x \in I^{1}$ such that $g^{N \cdot M}(x)=x$ and $g^{n}(x) \in I^{n+1}$ for every $n=1, \ldots, N \cdot M-1$. Clearly, $x \in I_{1}$ and $f^{N}(x)=x$ and $f^{i}(x) \in I_{i+1}$ for every $i=1, \ldots, N-1$. Thus, $f \in \mathcal{I}$.

It was noticed in [8] that the property $\mathcal{I}$ of all connected $G_{\delta}$ functions implies the property $\mathcal{I}$ of all their compositions. Since Theorem 3.4 is a consequence of the Darboux property and the property $\mathcal{I}$ of the function considered, it can be generalized to the class of all compositions of connected $G_{\delta}$ functions.

In $[8]$ it was also shown how to use the property $\mathcal{I}$ of the composition of connected $G_{\delta}$ functions to find a fixed point of the composition of finitely many derivatives - a generalization of results from [2] and [4].

3. The result. The proof of Sharkovskili's theorem for continuous functions, given by Block, Guckenheimer, Misiurewicz and Young and presented in [3], falls into several parts:

(i) every continuous function has the property $\mathcal{I}$;

(ii) the existence of a periodic point of an odd period $P$ implies the existence of a cycle $I_{1} \rightarrow \ldots \rightarrow I_{Q} \rightarrow I_{1}$ of closed intervals $I_{1}, \ldots, I_{Q}$ for each $Q \triangleleft P$;

(iii) the existence of a periodic point of an even period implies the existence of cycles $I_{1} \rightarrow I_{2} \rightarrow I_{1}$ and $I_{1} \rightarrow I_{1}$ of closed intervals $I_{1}, I_{2}$; 
(iv) the remaining cases reduce to the previous ones, because the class of continuous functions is closed under iterations.

We will show the analogous theorem for the class of all finite iterations of connected $G_{\delta}$ functions in the same way. First, we prove the analogue of (i) (Lemmas 3.1, 3.2 and Corollaries 1, 2). Then we notice that parts (ii) and (iii) of the proof in [3] work in our case (Lemma 3.3). Finally, we use the fact that the class of functions considered is closed under iterations.

The following lemma is rather technical - see Figures 3.A and 3.B for an illustration and note the symmetry between the assertion and assumption.

Lemma 3.1. Let $f: \mathbb{R} \rightarrow \mathbb{R}$ be a connectivity function, $N \in \mathbb{N}, n \in$ $\{1, \ldots, N\}$ and suppose $f \subset G$, where $G$ is an open subset of $\mathbb{R}^{2}$. Suppose that there exists a family $\left\{I_{k}\right\}_{1 \leq k \leq N}$ of bounded closed intervals and $y_{1}<y_{2}$ such that (counting naturals modulo $N$ ):

(1) there is no $y \in I_{n}$ such that $f^{N}(y)=y$ and $f^{i}(y) \in I_{n+i}$ for every $i=1, \ldots, N-1$

(2) $y_{1}, y_{2} \in I_{n}$;

(3) $f^{i}\left(y_{a}\right) \in I_{n+i}$ for every $i=1, \ldots, N-1$ and $a=1,2$;

(4) $\left[y_{1}, y_{2}\right] \subset\left[f^{N}\left(y_{1}\right), f^{N}\left(y_{2}\right)\right]$.

Then there exists a family $\left\{I_{k}^{\prime}\right\}_{1 \leq k \leq N}$ of closed intervals with $I_{i}^{\prime} \subset I_{i}$ for each $i, I_{n-1}^{\prime} \times I_{n}^{\prime} \subset G$ and $x_{1}^{\prime}<x_{2}^{\prime}$ such that:

$\left(1^{\prime}\right)$ there is no $x \in I_{n-1}^{\prime}$ such that $f^{N}(x)=x$ and $f^{i}(x) \in I_{n-1+i}^{\prime}$ for every $i=1, \ldots, N-1$;

$\left(2^{\prime}\right) x_{1}^{\prime}, x_{2}^{\prime} \in I_{n-1}^{\prime}$;

$\left(3^{\prime}\right) f^{i}\left(x_{a}^{\prime}\right) \in I_{n-1+i}^{\prime}$ for every $i=1, \ldots, N-1$ and $a=1,2$;

$\left(4^{\prime}\right)\left[x_{1}^{\prime}, x_{2}^{\prime}\right] \subset\left[f^{N}\left(x_{1}^{\prime}\right), f^{N}\left(x_{2}^{\prime}\right)\right]$.

Proof. For every function $h: \mathbb{R} \rightarrow \mathbb{R}$ define

$$
\begin{aligned}
& A(h)=\left\{\langle x, f(x)\rangle \in \mathbb{R}^{2} \mid x<h(f(x))\right\}, \\
& B(h)=\left\{\langle x, f(x)\rangle \in \mathbb{R}^{2} \mid x>h(f(x))\right\} .
\end{aligned}
$$

Clearly $A(h) \cap B(h)=\emptyset$. Moreover, if $f \cap h^{-1}=\emptyset$, then $f=A(h) \cup B(h)$.

For every $i=1, \ldots, N$ define $f_{i}:\left[f^{i-1}\left(y_{1}\right), f^{i-1}\left(y_{2}\right)\right] \rightarrow\left[f^{i}\left(y_{1}\right), f^{i}\left(y_{2}\right)\right]$ by

$$
f_{i}(x)= \begin{cases}\min \left\{f^{i}\left(y_{1}\right), f^{i}\left(y_{2}\right)\right\} & \text { if } f(x)<\min \left\{f^{i}\left(y_{1}\right), f^{i}\left(y_{2}\right)\right\}, \\ \max \left\{f^{i}\left(y_{1}\right), f^{i}\left(y_{2}\right)\right\} & \text { if } f(x)>\max \left\{f^{i}\left(y_{1}\right), f^{i}\left(y_{2}\right)\right\} \\ f(x) & \text { otherwise. }\end{cases}
$$

The functions $f_{i}$ are used to define $g: \mathbb{R} \rightarrow \mathbb{R}$ below. If $f^{N-1}\left(y_{1}\right)<f^{N-1}\left(y_{2}\right)$, 


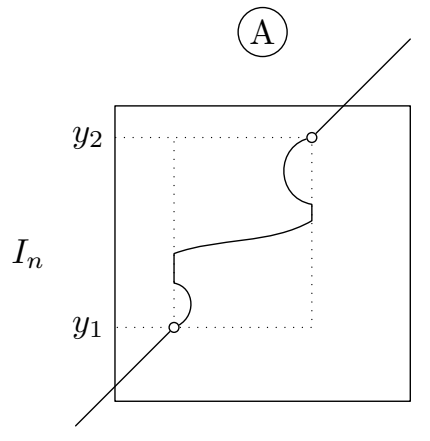

$I_{n-1}$

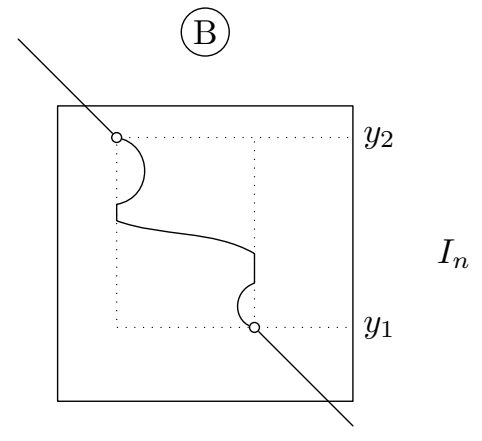

$I_{n-1}$

Fig. 1. The set $g^{-1}$. The points denoted by $\circ$ are from $\left(f^{N-1}\right)^{-1}$

we set (see Fig. 1.A)

$$
g(x)= \begin{cases}x-y_{1}+f^{N-1}\left(y_{1}\right) & \text { if } x<y_{1}, \\ \left(f_{N-1} \circ \ldots \circ f_{2} \circ f_{1}\right)(x) & \text { if } x \in\left[y_{1}, y_{2}\right], \\ x-y_{2}+f^{N-1}\left(y_{2}\right) & \text { if } x>y_{2},\end{cases}
$$

while if $f^{N-1}\left(y_{1}\right)>f^{N-1}\left(y_{2}\right)$, we define (see Fig. 1.B)

$$
g(x)= \begin{cases}y_{1}-x+f^{N-1}\left(y_{1}\right) & \text { if } x<y_{1}, \\ \left(f_{N-1} \circ \ldots \circ f_{2} \circ f_{1}\right)(x) & \text { if } x \in\left[y_{1}, y_{2}\right], \\ y_{2}-x+f^{N-1}\left(y_{2}\right) & \text { if } x>y_{2} .\end{cases}
$$

Note that $g$ is a Darboux function such that

$$
f^{N-1}\left(y_{1}\right)=g\left(y_{1}\right), \quad f^{N-1}\left(y_{2}\right)=g\left(y_{2}\right) .
$$

Moreover, if $g(y) \in\left(g\left(y_{1}\right), g\left(y_{2}\right)\right)$ for some $y \in\left[y_{1}, y_{2}\right]$, then

$$
g(y)=f^{N-1}(y)
$$

and by assumption (3),

$$
f^{i}(y) \in I_{n+i} \quad \text { for every } i=1, \ldots, N-1 .
$$

From Remark 2.2 and assumptions (1), (4) we have

$$
\left(f\left\lceil\left[g\left(y_{1}\right), g\left(y_{2}\right)\right]\right) \cap g^{-1}=\emptyset .\right.
$$

Note also that by (4), either $f^{N}\left(y_{1}\right)<y_{1}$ and $f^{N}\left(y_{2}\right)>y_{2}$, or $f^{N}\left(y_{1}\right)>y_{2}$ and $f^{N}\left(y_{2}\right)<y_{1}$. So, depending on the construction of $g$, we have either $\left\langle g\left(y_{1}\right), f\left(g\left(y_{1}\right)\right)\right\rangle \in A(g)$ and $\left\langle g\left(y_{2}\right), f\left(g\left(y_{2}\right)\right)\right\rangle \in B(g)$, or $\left\langle g\left(y_{1}\right), f\left(g\left(y_{1}\right)\right)\right\rangle \in$ $B(g)$ and $\left\langle g\left(y_{2}\right), f\left(g\left(y_{2}\right)\right)\right\rangle \in A(g)$. It follows that

$$
\left\{\begin{array}{l}
f\left\lceil\left[g\left(y_{1}\right), g\left(y_{2}\right)\right] \cap\left(\mathbb{R} \times\left(-\infty, y_{1}\right]\right) \subset A(g),\right. \\
f \uparrow\left[g\left(y_{1}\right), g\left(y_{2}\right)\right] \cap\left(\mathbb{R} \times\left[y_{2}, \infty\right)\right) \subset B(g)
\end{array}\right.
$$


if $\left\langle g\left(y_{1}\right), f\left(g\left(y_{1}\right)\right)\right\rangle \in A(g)$ and $\left\langle g\left(y_{2}\right), f\left(g\left(y_{2}\right)\right)\right\rangle \in B(g)$, while

$$
\left\{\begin{array}{l}
f \uparrow\left[g\left(y_{1}\right), g\left(y_{2}\right)\right] \cap\left(\mathbb{R} \times\left(-\infty, y_{1}\right]\right) \subset B(g), \\
f \uparrow\left[g\left(y_{1}\right), g\left(y_{2}\right)\right] \cap\left(\mathbb{R} \times\left[y_{2}, \infty\right)\right) \subset A(g)
\end{array}\right.
$$

if $\left\langle g\left(y_{1}\right), f\left(g\left(y_{1}\right)\right)\right\rangle \in B(g)$ and $\left\langle g\left(y_{2}\right), f\left(g\left(y_{2}\right)\right)\right\rangle \in A(g)$.

By Remark 2.1 there exists an $x^{\prime} \in\left[g\left(y_{1}\right), g\left(y_{2}\right)\right]$ such that for every open neighbourhood $U$ of $\left\langle x^{\prime}, f\left(x^{\prime}\right)\right\rangle$, both $U \cap A(g) \neq \emptyset$ and $U \cap B(g) \neq \emptyset$.

First, we show that $f\left(x^{\prime}\right) \in\left[y_{1}, y_{2}\right]$. Indeed, because $g$ is continuous outside $\left[y_{1}, y_{2}\right]$, for every $x \in\left[g\left(y_{1}\right), g\left(y_{2}\right)\right]$ such that $f(x) \notin\left[y_{1}, y_{2}\right]$ there exists an open neighbourhood $V$ of $\langle x, f(x)\rangle$ such that $f \cap V \subset A(g)$ if $\langle x, f(x)\rangle \in A(g)$, while $f \cap V \subset B(g)$ if $\langle x, f(x)\rangle \in B(g)$.

Next, observe that if we set

$$
A_{0}(g)=A(g) \cap\left(\mathbb{R} \times\left[y_{1}, y_{2}\right]\right), \quad B_{0}(g)=B(g) \cap\left(\mathbb{R} \times\left[y_{1}, y_{2}\right]\right),
$$

and let $U$ be an open neighbourhood of $\left\langle x^{\prime}, f\left(x^{\prime}\right)\right\rangle$, then $U \cap A_{0}(g) \neq \emptyset$ and $U \cap B_{0}(g) \neq \emptyset$. This is a consequence of inclusions $(\alpha)$ and $(\beta)$.

Take closed intervals $I_{n-1}^{\prime} \subset I_{n-1}$ and $I_{n}^{\prime} \subset I_{n}$ such that:

- $\left\langle x^{\prime}, f\left(x^{\prime}\right)\right\rangle \in I_{n-1}^{\prime} \times I_{n}^{\prime}$,

- $I_{n-1}^{\prime} \times I_{n}^{\prime} \subset G$,

- $\left\langle g\left(f\left(x^{\prime}\right)\right), f\left(x^{\prime}\right)\right\rangle \notin I_{n-1}^{\prime} \times I_{n}^{\prime}$,

- if $U$ is an open neighbourhood of $\left\langle x^{\prime}, f\left(x^{\prime}\right)\right\rangle$, then $U \cap A_{0}(g) \cap\left(I_{n-1}^{\prime} \times I_{n}^{\prime}\right)$ $\neq \emptyset$ and $U \cap B_{0}(g) \cap\left(I_{n-1}^{\prime} \times I_{n}^{\prime}\right) \neq \emptyset$.

For every $k=1, \ldots, n-2, n+1, \ldots, N$ take $I_{k}^{\prime}=I_{k}$.

Without loss of generality we can assume that $\left\langle x^{\prime}, f\left(x^{\prime}\right)\right\rangle \in A_{0}(g)$, i.e. $x^{\prime}<g\left(f\left(x^{\prime}\right)\right)$ (the other case is symmetric). Fix $\langle p, f(p)\rangle \in B_{0}(g) \cap\left(I_{n-1}^{\prime} \times I_{n}^{\prime}\right)$. Since $x^{\prime}<g\left(f\left(x^{\prime}\right)\right) \notin I_{n-1}^{\prime}$, it follows that

$$
\left\{x \in I_{n-1}^{\prime} \mid f(x)=f\left(x^{\prime}\right)\right\} \subset A(g) .
$$

Hence $f(p) \neq f\left(x^{\prime}\right)$. Two cases are possible:

1. $p<x^{\prime}$

2. $p>x^{\prime}$.

Case 1 (see Fig. 2.A). By the Darboux property of $g$, there exist $q_{1}, q_{2} \in$ $\left(f(p), f\left(x^{\prime}\right)\right)$ such that

$$
\min \left\{g\left(y_{1}\right), g\left(y_{2}\right)\right\}<g\left(q_{1}\right)<p, \quad \max \left\{g\left(y_{1}\right), g\left(y_{2}\right)\right\}>g\left(q_{2}\right)>x^{\prime} .
$$

Since $f$ is Darboux, there exist $p_{1}^{\prime}, p_{2}^{\prime} \in\left(p, x^{\prime}\right)$ such that $f\left(p_{1}^{\prime}\right)=q_{1}$ and $f\left(p_{2}^{\prime}\right)=q_{2}$. Let $x_{1}^{\prime}=\min \left\{p_{1}^{\prime}, p_{2}^{\prime}\right\}$ and $x_{2}^{\prime}=\max \left\{p_{1}^{\prime}, p_{2}^{\prime}\right\}$.

Since $g\left(f\left(x_{1}^{\prime}\right)\right), g\left(f\left(x_{2}^{\prime}\right)\right) \in\left(g\left(y_{1}\right), g\left(y_{2}\right)\right)$, it follows that $f^{i}\left(f\left(x_{a}^{\prime}\right)\right) \in I_{n+i}$ for every $i=1, \ldots, N-1$ and $a=1,2$. Therefore $f^{i+1}\left(x_{a}^{\prime}\right) \in I_{n+i}^{\prime}$ for every $i=0, \ldots, N-2$ and $a=1,2$. So, $x_{1}^{\prime}$ and $x_{2}^{\prime}$ are as required. 
(A)

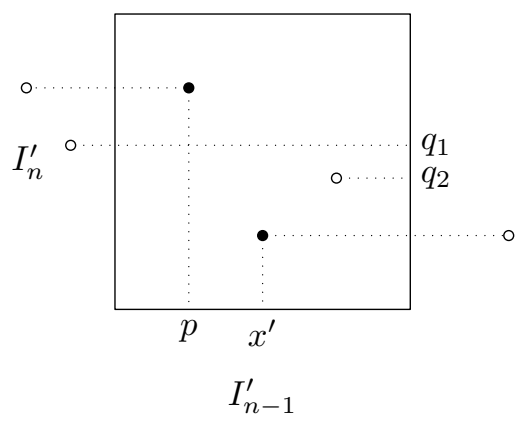

(B)

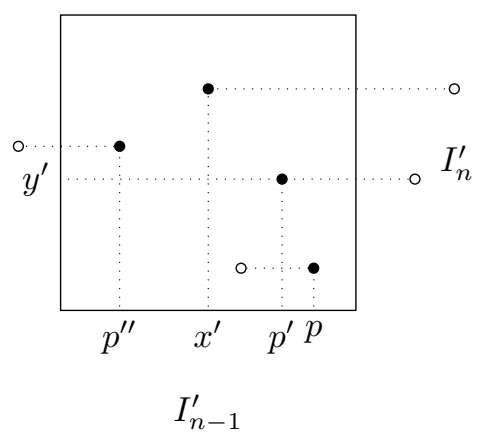

Fig. 2. Configurations from the proof of Lemma 3.1. The points denoted by $\circ$ are from $g^{-1}$, those denoted by $\bullet$ are from $f$

CASE 2 (see Fig. 2.B). Since $g$ is bilaterally dense in $f\left(x^{\prime}\right)$, there exists $y^{\prime} \in\left(f\left(x^{\prime}\right), f(p)\right)$ such that $g\left(y^{\prime}\right)>p$. By the Darboux property of $f$ there exists $p^{\prime} \in\left(x^{\prime}, p\right)$ such that $f\left(p^{\prime}\right)=y^{\prime}$. We can also find $\left\langle p^{\prime \prime}, f\left(p^{\prime \prime}\right)\right\rangle \in$ $B_{0}(g) \cap\left(I_{n-1}^{\prime} \times I_{n}^{\prime}\right)$ with $p^{\prime \prime}<p^{\prime}$.

As in the previous case, by the Darboux property of $g$ there exist $q_{1}, q_{2} \in$ $\left(f\left(p^{\prime}\right), f\left(p^{\prime \prime}\right)\right)$ such that

$$
\min \left\{g\left(y_{1}\right), g\left(y_{2}\right)\right\}<g\left(q_{1}\right)<p^{\prime}, \quad \max \left\{g\left(y_{1}\right), g\left(y_{2}\right)\right\}>g\left(q_{2}\right)>p^{\prime \prime} .
$$

From the Darboux property of $f$ there exist $p_{1}^{\prime}, p_{2}^{\prime} \in\left(p^{\prime \prime}, p^{\prime}\right)$ such that $f\left(p_{1}^{\prime}\right)=q_{1}$ and $f\left(p_{2}^{\prime}\right)=q_{2}$. Let $x_{1}^{\prime}=\min \left\{p_{1}^{\prime}, p_{2}^{\prime}\right\}$ and $x_{2}^{\prime}=\max \left\{p_{1}^{\prime}, p_{2}^{\prime}\right\}$.

Since $g\left(f\left(x_{1}^{\prime}\right)\right), g\left(f\left(x_{2}^{\prime}\right)\right) \in\left(g\left(y_{1}\right), g\left(y_{2}\right)\right)$, it follows that $f^{i}\left(f\left(x_{a}^{\prime}\right)\right) \in I_{n+i}$ for every $i=1, \ldots, N-1$ and $a=1,2$. Therefore $f^{i+1}\left(x_{a}^{\prime}\right) \in I_{n+i}^{\prime}$ for every $i=0, \ldots, N-2, a=1,2$. So, $x_{1}^{\prime}$ and $x_{2}^{\prime}$ are as desired.

Lemma 3.2. Suppose that $f: \mathbb{R} \rightarrow \mathbb{R}$ is a connectivity function, $N \in \mathbb{N}$ and $\left\{I_{k}\right\}_{1 \leq k \leq N}$ is a family of closed intervals such that $I_{1} \rightarrow \ldots \rightarrow I_{N} \rightarrow I_{1}$. If $G$ is an open set such that $f \subset G$, then there exists a family $\left\{J_{k}\right\}_{1 \leq k \leq N}$ of closed intervals with $J_{i} \subset I_{i}$ and $J_{i} \times J_{i+1} \subset G$ for every $i=1, \ldots, N$ such that $J_{1} \rightarrow \ldots \rightarrow J_{N} \rightarrow J_{1}$.

Proof. First note that if there exists an $x \in I_{1}$ such that $f^{N}(x)=x$ and $f^{i}(x) \in I_{i+1}$ for $i=1, \ldots, N-1$, then the family $\left\{J_{k}\right\}_{1 \leq k \leq N}$ of degenerate intervals $J_{k}=\left\{f^{k-1}(x)\right\}$ is as desired. So, we assume that there is no such $x$. (This implies in particular that all intervals $I_{i}$ are non-degenerate.)

Take $p, q$ such that $p<q$ and $I_{1}=[p, q]$. Since $f$ is Darboux and $f\left(I_{N}\right) \supset$ $I_{1}$, there exist $x_{p}^{N}, x_{q}^{N} \in I_{N}$ such that $f\left(x_{p}^{N}\right)=p$ and $f\left(x_{q}^{N}\right)=q$. Again, since $f\left(I_{N-1}\right) \supset I_{N}$, there exist $x_{p}^{N-1}, x_{q}^{N-1} \in I_{N-1}$ such that $f\left(x_{p}^{N-1}\right)=x_{p}^{N}$ and 
$f\left(x_{q}^{N-1}\right)=x_{q}^{N}$. Inductively, we can find a sequence $\left\{x_{p}^{k}, x_{q}^{k}\right\}_{1 \leq k \leq N}$ of pairs such that $f\left(x_{p}^{N}\right)=p, f\left(x_{q}^{N}\right)=q$ and for each $i=1, \ldots, N-1$ :

- $x_{p}^{i}, x_{q}^{i} \in I_{i}$,

- $f\left(x_{p}^{i}\right)=x_{p}^{i+1}$ and $f\left(x_{q}^{i}\right)=x_{q}^{i+1}$.

Now, if we set

$$
y_{1}=\min \left\{x_{p}^{1}, x_{q}^{1}\right\}, \quad y_{2}=\max \left\{x_{p}^{1}, x_{q}^{1}\right\},
$$

then $f^{i}\left(y_{1}\right) \in I_{i+1}$ and $f^{i}\left(y_{2}\right) \in I_{i+1}$ for every $i=1, \ldots, N$. Since $y_{1}, y_{2} \in I_{1}$ and either $f^{N}\left(y_{1}\right)=p$ and $f^{N}\left(y_{2}\right)=q$, or $f^{N}\left(y_{1}\right)=q$ and $f^{N}\left(y_{2}\right)=p$, it follows that $\left[y_{1}, y_{2}\right] \subset[p, q]=\left[f^{N}\left(y_{1}\right), f^{N}\left(y_{2}\right)\right]$.

Note that $G, N, f, y_{1}, y_{2}$ and $\left\{I_{k}\right\}_{1 \leq k \leq N}$ satisfy all the assumptions of Lemma 3.1 with $n=1$ (see Fig. 3.A). So, there exists a family $\left\{I_{k}^{\prime}\right\}_{1 \leq k \leq N}$ and $x_{1}^{\prime}, x_{2}^{\prime}$ with $I_{N}^{\prime} \times I_{1}^{\prime} \subset G$ as in the assertion of that lemma (see Fig. $3 . \bar{B}$ ). Since $f^{N-1}\left(x_{1}^{\prime}\right) \in I_{N-1}^{\prime}$ and $f^{N-1}\left(x_{2}^{\prime}\right) \in I_{N-1}^{\prime}$ (see Fig. 3.C), $G, N, f, x_{1}^{\prime}, x_{2}^{\prime}$ and $\left\{I_{k}^{\prime}\right\}_{1 \leq k \leq N}$ again satisfy all the assumptions of Lemma 3.1 with $n$ decreased by 1 (recall that we count naturals modulo $N$, so in this step $n=N$; see Fig. 3.D).

If we repeat this operation $N$ times, we obtain $z_{1}, z_{2} \in \mathbb{R}$ and a family $\left\{I_{k}^{\prime \prime}\right\}_{1 \leq k \leq N}$ of intervals such that:

(1) $I_{i}^{\prime \prime} \subset I_{i}$ and $I_{i}^{\prime \prime} \times I_{i+1}^{\prime \prime} \subset G$ for every $i=1, \ldots, N$;

(2) $z_{1}, z_{2} \in I_{1}^{\prime \prime}$

(3) $f^{i}\left(z_{a}\right) \in I_{i+1}^{\prime \prime}$ for every $i=1, \ldots, N-1$ and $a=1,2$;

(4) $\left[z_{1}, z_{2}\right] \subset\left[f^{N}\left(z_{1}\right), f^{N}\left(z_{2}\right)\right]$.

Now we can define the desired family $\left\{J_{k}\right\}_{1 \leq k \leq N}$ as follows:

- $J_{1}=\left[z_{1}, z_{2}\right]$

- $J_{i}=\left[f^{i-1}\left(z_{1}\right), f^{i-1}\left(z_{2}\right)\right]$ for $i=2, \ldots, N$.

By the Darboux property of $f, J_{i} \rightarrow J_{i+1}$ for every $i=1, \ldots, N-1$. Moreover, from (4) we have $J_{N} \rightarrow J_{1}$. Items (2) and (3) give $J_{i} \subset I_{i}^{\prime \prime}$ for each $i$, so by (1), $J_{i} \subset I_{i}$ and $J_{i} \times J_{i+1} \subset G$.

The continuous version of the following corollary is known in one dimensional dynamics as the "Itinerary Lemma".

Corollary 1. Every connectivity $G_{\delta}$ function $f: \mathbb{R} \rightarrow \mathbb{R}$ has the property $\mathcal{I}$.

Proof. Let $N \in \mathbb{N}$ and $\left\{I_{k}\right\}_{1 \leq k \leq N}$ be a family of closed intervals such that $I_{1} \rightarrow \ldots \rightarrow I_{N} \rightarrow I_{1}$.

Since $f$ is a $G_{\delta}$ subset of $\mathbb{R}^{2}$, there exists a family $\mathcal{G}=\left\{G_{n}\right\}_{n \in \mathbb{N}}$ of open sets such that $f=\bigcap \mathcal{G}$.

Using Lemma 3.2 we can find a family $\mathcal{J}_{1}=\left\{J_{k}^{1}\right\}_{1 \leq k \leq N}$ of closed intervals such that for every $i=1, \ldots, N$ : 


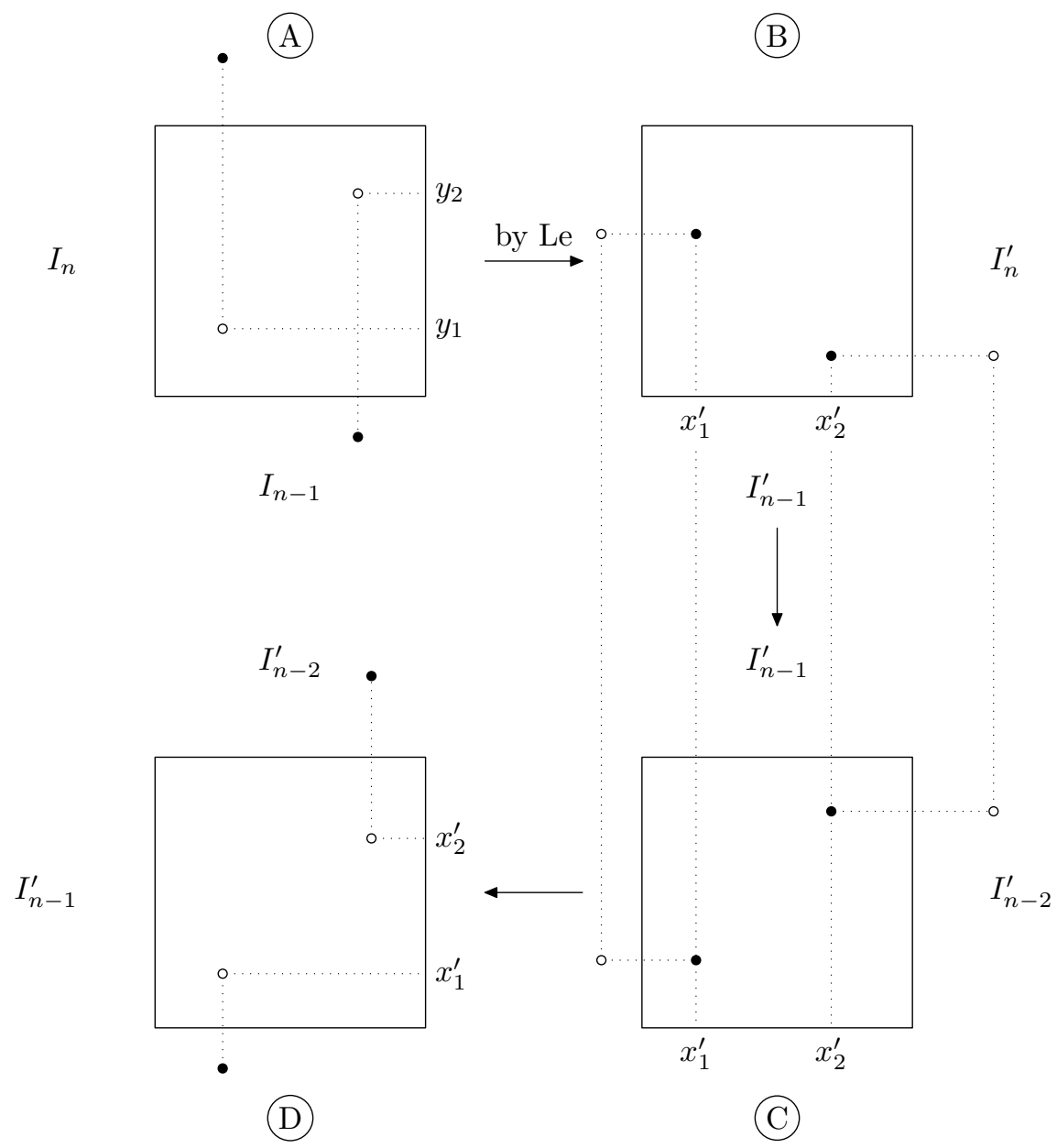

Fig. 3. Using Lemma 3.1 to build a sequence of decreasing rectangles. Configuration "A" is as in the assumption of the lemma. The points denoted by $\circ$ are from $\left(f^{N-1}\right)^{-1}$, those denoted by $\bullet$ are from $f$. Configuration "B" illustrates the assertion of lemma. Configuration "C" is a redrawing of "B", with the •'s from $f^{N-1}$ and the o's from $f^{-1}$. (Note that by $\left(3^{\prime}\right), f^{N-1}\left(x_{a}^{\prime}\right) \in I_{n-2}^{\prime}$ for $a=1,2$.) Configuration " $\mathrm{D}$ " is "C" with axes swapped. Again, the o's are from $\left(f^{N-1}\right)^{-1}$, and the $\bullet$ 's are from $f$. Configurations "A" and "D" are symmetric - with " $n$ ", " $y$ " and " $I$ " translated to " $n-1$ ", " $x$ " and " $I$ ", respectively. So, "D" again satisfies all the assumptions of Lemma 3.1.

- $J_{i}^{1} \subset I_{i}$

- $J_{i}^{1} \times J_{i+1}^{1} \subset G_{1}$;

- $J_{1}^{1} \rightarrow J_{2}^{1} \rightarrow \ldots \rightarrow J_{N}^{1} \rightarrow J_{1}^{1}$.

Continuing inductively we can build a sequence $\left\{\mathcal{J}_{s}\right\}_{s \in \mathbb{N}}$ of families of closed intervals with $\mathcal{J}_{s}=\left\{J_{k}^{s}\right\}_{1 \leq k \leq N}$ such that for every $s \in \mathbb{N}$ and $i=1, \ldots, N$ : 
- $J_{i}^{s+1} \subset J_{i}^{s}$

- $J_{i}^{s} \times J_{i+1}^{s} \subset G_{s}$;

- $J_{1}^{s} \rightarrow J_{2}^{s} \rightarrow \ldots \rightarrow J_{N}^{s} \rightarrow J_{1}^{s}$.

It follows that for every $i=1, \ldots, N$ there exists an $x_{i} \in \bigcap_{s \in \mathbb{N}} J_{i}^{s}$. Obviously, $x_{i} \in I_{i}$. Since $J_{i}^{s} \times J_{i+1}^{s} \subset G_{s}$ for every $s \in \mathbb{N}$, we have $\left\langle x_{i}, x_{i+1}\right\rangle \in$ $\bigcap \mathcal{G}=f$. So, $f\left(x_{i}\right)=x_{i+1}$ and if we take $x=x_{1}$, then $f^{N}(x)=x$ and $f^{i}(x) \in I_{i+1}$. Thus, $f \in \mathcal{I}$.

From Remark 2.4 we get a stronger fact (see Example 4.1):

Corollary 2. Suppose that $M \in \mathbb{N}$ and $f=g^{M}$, where $g: \mathbb{R} \rightarrow \mathbb{R}$ is a connected $G_{\delta}$ subset of $\mathbb{R}^{2}$. Then $f$ has the property $\mathcal{I}$.

The next lemma is extracted from the proof of Sharkovski1's theorem presented in [3]. The whole proof works only for the continuous case, but this part does not need the full strength of this assumption. For completeness, we repeat the argument (with slight modifications) in the Appendix.

Lemma 3.3. Suppose that $f: \mathbb{R} \rightarrow \mathbb{R}$ is a Darboux function with the property $\mathcal{I}$. If $P \in \mathbb{N}$ and $f$ has a periodic point of prime period $P$, then:

(1) if $P>1$ and $P$ is odd, then for every $Q>P$, and for every even $Q$, and for $Q=1$ there exist closed intervals $I_{1}, \ldots, I_{Q}$ such that $I_{1} \rightarrow_{f} \ldots \rightarrow_{f}$ $I_{Q} \rightarrow_{f} I_{1}$, and moreover, for every $x \in I_{1}$ such that $f^{Q}(x)=x$ and $f^{i}(x) \in$ $I_{i+1}$ for $i=1, \ldots, Q-1, x$ is of prime period $Q$ for $f$

(2) if $P>2$ and $P$ is even, then there exist closed intervals $I_{1}$ and $I_{2}$ such that $I_{1} \rightarrow_{f} I_{1}$ and $I_{1} \rightarrow_{f} I_{2} \rightarrow_{f} I_{1}$, and moreover, for every $x \in I_{1}$ such that $f^{2}(x)=x$ and $f(x) \in I_{2}, f(x) \neq x$;

(3) if $P=2$, then there exists a closed interval $I_{1}$ such that $I_{1} \rightarrow_{f} I_{1}$.

Theorem 3.4. Suppose that $M \in \mathbb{N}$ and $f=g^{M}$, where $g: \mathbb{R} \rightarrow \mathbb{R}$ is a connected $G_{\delta}$ subset of $\mathbb{R}^{2}$. If $f$ has a point of prime period $P$ and $Q \triangleleft P$, then $f$ also has a point of prime period $Q$.

Proof. First, suppose that $P>1$ is odd and $Q>P$ (or $Q$ is even, or $Q=1$ ). By Lemma 3.3(1) there exist closed intervals $I_{1}, \ldots, I_{Q}$ such that $I_{1} \rightarrow_{f} \ldots \rightarrow_{f} I_{Q} \rightarrow_{f} I_{1}$. Now, by Corollary 2 there exists an $x \in I_{1}$ such that $f^{Q}(x)=x$ and $f^{i}(x) \in I_{i}$ for every $i=1, \ldots, Q$. By the "moreover" part of Lemma 3.3(1), $x$ is a point of prime period $Q$ for $f$.

Next, suppose that $P$ is even. If $P>2$, then by Lemma 3.3(2) and Corollary $2, f$ has a fixed point and a point of prime period 2. If $P=2$, then by Lemma 3.3(3) and Corollary $2, f$ has a fixed point.

Obviously, the class of finite iterations of connectivity $G_{\delta}$ functions is closed under finite iterations. So, we can use standard arguments from the proof of the continuous case. 
Suppose that $P=2^{a}$ and $Q=2^{b}$, where $b<a$. Consider $h=f^{Q / 2}$. Since $h$ has a point of prime period $2^{a-b+1}$, by the observation above it also has a point of prime period 2. This point has prime period $2^{b}$ for $f$.

Finally, suppose that $P=p \cdot 2^{a}$, where $p$ is odd. By the method presented above, this case can be reduced to the previous ones.

The following corollary answers the question from [7].

COROLlary 3. The conclusion of Sharkovskiu's theorem holds for derivatives and $\mathrm{DB}_{1}$ real functions.

4. Problems. A natural problem suggested by the formulation of Theorem 3.4 is to get a nice characterization of the class of "finite iterations of connected $G_{\delta}$ real functions" or "finite compositions of connected $G_{\delta}$ real functions" (see also the problem from [6]: "characterize compositions of two derivatives ( $\mathrm{DB}_{1}$ functions)"). We will modify Example 2.2 from [1] to show that an iteration of a connected $G_{\delta}$ function need not be $G_{\delta}$.

EXAMPLE 4.1. There exists a connectivity $G_{\delta}$ function $f: \mathbb{R} \rightarrow \mathbb{R}$ such that the graph of $f^{2}$ is not a $G_{\delta}$ subset of $\mathbb{R}^{2}$.

Proof. Let $C \subset[0,1]$ be the Cantor ternary set with $\left\{\left(a_{n}, b_{n}\right)\right\}_{n \in \mathbb{N}}$ being all the components of its complement. Let $C_{0}=C \backslash \bigcup_{n \in \mathbb{N}}\left\{a_{n}, b_{n}\right\}$. Define $f$ as follows.

- For every $n \in \mathbb{N}$ take $m_{n} \in \mathbb{N}$ such that $\left(a_{m_{n}}+b_{m_{n}}\right) / 2 \in\left(1-2^{-(n+1)}, 1\right)$. Put $f\left(a_{n}\right)=0$ and $f\left(b_{n}\right)=\left(a_{m_{n}}+b_{m_{n}}\right) / 2$ and $f\left(\left(a_{n}+b_{n}\right) / 2\right)=1 / 2$. Extend it linearly onto $\left[a_{n}, b_{n}\right]$ to a strictly increasing continuous function.

- Put $f(x)=0$ elsewhere.

By the same arguments as in [1, Example 2.2], $f$ is a connectivity $G_{\delta}$ function. But since $f^{2}\left(a_{n}\right)=0, f^{2}\left(b_{n}\right)=1 / 2$ and $f^{2}\left\lceil C_{0}=0\right.$ for every $n \in \mathbb{N}$, it follows that $f^{2} \cap(C \times\{1 / 2\})$ is a countable dense subset of the Baire space $C \times\{1 / 2\}$, so it cannot be a $G_{\delta}$ subset of $C \times\{1 / 2\}$. Thus $f^{2}$ cannot be $G_{\delta}$.

Another related problem is whether the conclusion of Sharkovski1's theorem holds for all connected Baire class 2 real functions (the graph of a Baire class 2 function need not be $G_{\delta}$ ).

Appendix: Proof of Lemma 3.3. This proof, due to Block, Guckenheimer, Misiurewicz and Young and contained in [3], was given for $f$ continuous, but it works for every Darboux function with the property $\mathcal{I}$.

Proof of Lemma 3.3. First assume that $f$ has a periodic point $x$ of prime period $P$ with $P$ odd and $P>1$. Suppose that $P$ is minimal with such properties. Let $x_{1}, \ldots, x_{P}$ be all points from the orbit of $x$, numbered from 
left to right. Note that $f$ permutes the orbit. Clearly, $f\left(x_{i}\right) \neq x_{i}$ for each $i$, thus $f\left(x_{1}\right)>x_{1}$ and $f\left(x_{P}\right)<x_{P}$. So, we can choose the largest $i$ for which $f\left(x_{i}\right)>x_{i}$. Let $I_{1}$ be the interval $\left[x_{i}, x_{i+1}\right]$. Since $f\left(x_{i+1}\right)<x_{i+1}$, it follows that $f\left(x_{i+1}\right) \leq x_{i}$ and so $f\left(I_{1}\right) \supset I_{1}$. Therefore, $I_{1} \rightarrow I_{1}$.

Let a basic interval be any interval of the form $\left[x_{j}, x_{j+1}\right]$.

Since $x$ does not have period 2 , it cannot be that $f\left(x_{i+1}\right)=x_{i}$ and $f\left(x_{i}\right)=x_{i+1}$, so $f\left(I_{1}\right)$ contains at least one other basic interval (we will see below that there is exactly one such interval). Let $\mathcal{O}_{2}$ denote the family of all basic intervals which are $f$-covered by $I_{1}$. Hence $I_{1} \in \mathcal{O}_{2}$ but $\mathcal{O}_{2} \neq\left\{I_{1}\right\}$ and if $I_{2}$ is any interval in $\mathcal{O}_{2}$, then $I_{1} \rightarrow I_{2}$. It can be observed that $\bigcup \mathcal{O}_{2}$ is an interval itself.

Now let $\mathcal{O}_{3}$ denote the family of basic intervals which are $f$-covered by some interval in $\mathcal{O}_{2}$. Again, $\bigcup \mathcal{O}_{3}$ is an interval itself. Indeed, suppose $\bigcup \mathcal{O}_{3}$ is not connected. Then there exist $a, b \in \mathbb{N}$ with $1 \leq a<b \leq P$ and $\left[x_{a}, x_{b}\right] \cap \bigcup \mathcal{O}_{3}=\left\{x_{a}, x_{b}\right\}$. By the Darboux property of $f$, for every basic interval $\left[x_{j}, x_{j+1}\right] \in \mathcal{O}_{2}, f\left(x_{j}\right)$ and $f\left(x_{j+1}\right)$ are on the same side of $\left[x_{a}, x_{b}\right]$. But $\cup \mathcal{O}_{2}$ is connected and so all images of points $x_{j}$ from $\bigcup \mathcal{O}_{2}$ are on the same side of $\left[x_{a}, x_{b}\right]$. This is a contradiction, since there exist $a^{\prime}, b^{\prime} \in \bigcup \mathcal{O}_{2}$ such that $f\left(a^{\prime}\right)=x_{a}$ and $f\left(b^{\prime}\right)=x_{b}$.

Continuing inductively, let $\mathcal{O}_{l+1}$ be the family of basic intervals that are $f$-covered by some interval in $\mathcal{O}_{l}$. Note that for any interval $I_{l+1} \in \mathcal{O}_{l+1}$ there is a chain of intervals $I_{2}, \ldots, I_{l}$ with $I_{j} \in \mathcal{O}_{j}$ for each $j$ which satisfy $I_{1} \rightarrow I_{2} \rightarrow \ldots \rightarrow I_{l} \rightarrow I_{l+1}$.

Now $\left\{\mathcal{O}_{l}\right\}_{l \in \mathbb{N}}$ is an increasing sequence with $\bigcup \mathcal{O}_{l}$ being an interval for every $l \in \mathbb{N}$. Since there are only finitely many $x_{j}$, there is an $l$ for which $\mathcal{O}_{l+1}=\mathcal{O}_{l}$. For this $l, \mathcal{O}_{l}$ must contain all basic intervals, for otherwise $x$ would have period less than $P$.

We claim that there is at least one basic interval different from $I_{1}$ in some $\mathcal{O}_{k}$ which $f$-covers $I_{1}$. This follows since there are more points $x_{j}$ on one side of $I_{1}$ than on the other ( $P$ is odd). Hence there is an $x_{j}$ changing side under the action of $f$, and there is an $x_{j}$ which stays on the same side of $I_{1}$. Consequently, there is at least one interval which $f$-covers $I_{1}$.

Now consider chains of basic intervals $I_{1} \rightarrow I_{2} \rightarrow \ldots \rightarrow I_{k} \rightarrow I_{1}$ where $I_{2} \neq I_{1}$. By the above observations, there is at least one such chain. Choose one with the smallest $k$, i.e. $I_{1} \rightarrow I_{2} \rightarrow \ldots \rightarrow I_{k} \rightarrow I_{1}$ is the shortest path from $I_{1}$ to $I_{1}$ except, of course, $I_{1} \rightarrow I_{1}$. We therefore find a diagram as in Figure 4.A.

Clearly, $k \leq P-1$ (there are only $P-1$ basic intervals). Now, if $k<P-1$, then by the $\mathcal{I}$ property of $f$, one of the loops $I_{1} \rightarrow \ldots \rightarrow I_{k} \rightarrow I_{1}$ or $I_{1} \rightarrow \ldots \rightarrow I_{k} \rightarrow I_{1} \rightarrow I_{1}$ gives a fixed point of $f^{m}$ with $m$ odd and $m<P$. This point must have prime period odd and less than $P$ (and not equal to 1 , 
(A)

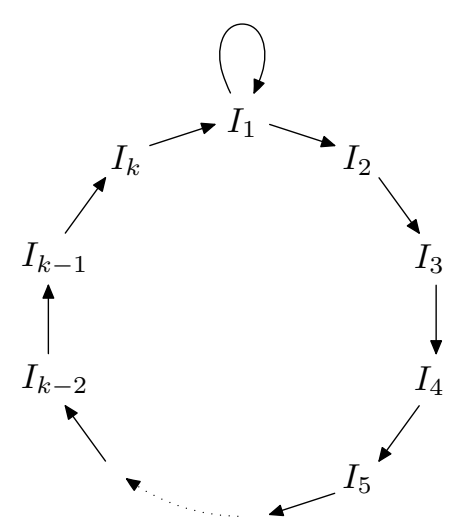

(B)

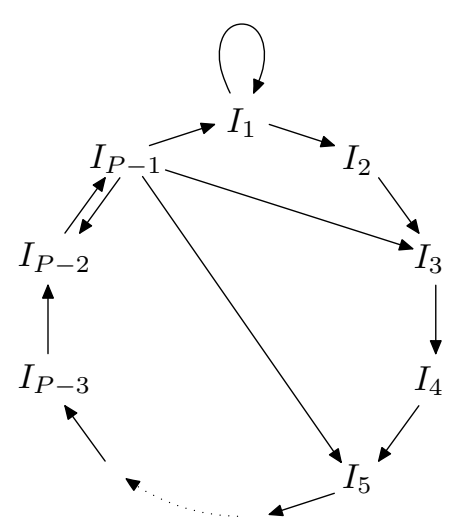

Fig. 4. An example of the shortest non-trivial path $I_{1} \rightarrow \ldots \rightarrow I_{1}$

since $I_{1} \cap I_{2}$ consists of at most one point, and that point has period $P$ ). This contradicts the minimality of $P$, so $k=P-1$.

By the minimality of $k$, we cannot have $I_{l} \rightarrow I_{j}$ for any $j>l+1$. So, the orbit of $x$ must be ordered in $\mathbb{R}$ in one of two possible ways, as depicted in Figure 5. It follows that we can extend the diagram from Figure 4.A to that shown in Figure 4.B.

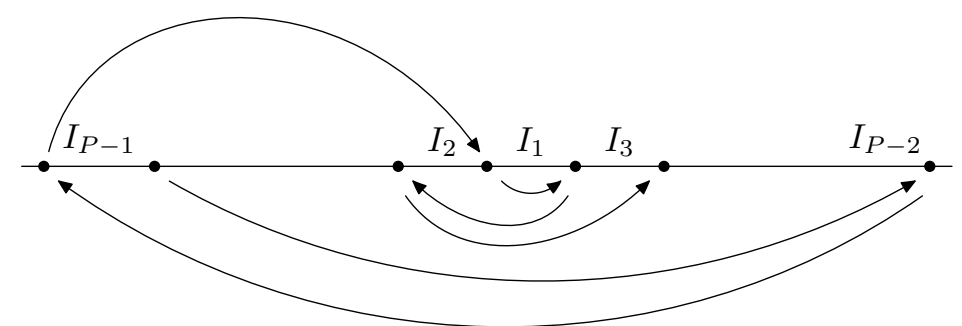

Fig. 5. An ordering of the shortest path. The other is its mirror image

The assertion for the case of $P$ odd is now immediate. Cycles of length greater than $P$ take the form

$$
I_{1} \rightarrow \ldots \rightarrow I_{P-1} \rightarrow I_{1} \rightarrow \ldots \rightarrow I_{1} .
$$

Cycles of smaller even length are given by paths of the form

$$
I_{P-1} \rightarrow I_{P-2} \rightarrow I_{P-1}, \quad I_{P-1} \rightarrow I_{P-4} \rightarrow I_{P-3} \rightarrow I_{P-2} \rightarrow I_{P-1}
$$

and so forth. A cycle of length 1 is given by the trivial path $I_{1} \rightarrow I_{1}$.

Note that all such cycles satisfy the second part of the assertion. This is a consequence of the fact that in every such path there is an interval with interior disjoint from all the other intervals. It follows that the prime period 
of every periodic point $y \neq x$ such that $f^{n-1}(y)$ lies in the $n$th interval of the cycle must be a multiple of the length of the cycle.

The case of $P$ even and greater than 2 follows from the above arguments provided we can guarantee that some points $x_{j}$ change sides under $f$ and some do not (use the facts that $I_{P-2} \rightarrow I_{P-1}$ and $I_{P-1} \rightarrow I_{P-2}$ ). If this is not the case, then every $x_{j}$ must change side and so

$$
\left[x_{1}, x_{i}\right] \rightarrow\left[x_{i+1}, x_{P}\right] \rightarrow\left[x_{1}, x_{i}\right]
$$

The case of $P=2$ is obvious, since

$$
\left[x_{1}, x_{2}\right] \rightarrow\left[x_{1}, x_{2}\right]
$$

\section{References}

[1] K. Ciesielski and H. Rosen, Two examples concerning extendable and almost continuous functions, Real Anal. Exchange 25 (1999-2000), 579-598.

[2] M. Csörnyei, T. C. O'Neil, and D. Preiss, The composition of two derivatives has a fixed point, ibid. 26 (2000-2001), 749-760.

[3] R. L. Devaney, An Introduction to Chaotic Dynamical Systems, Benjamin-Cummings, Menlo Park, 1986.

[4] M. Elekes, T. Keleti, and V. Prokaj, The fixed point of the composition of derivatives, Real Anal. Exchange 27 (2001-2002), 131-140.

[5] R. Gibson and T. Natkaniec, Darboux like functions, ibid. 22 (1996-1997), 492-533.

[6] - Darboux like functions. Old problems and new results, ibid. 24 (1998-1999), 487496.

[7] K. Kellum, Iterates of almost continuous functions and Sarkovskii's theorem, ibid. 14 (1988-1989), 420-422.

[8] P. Szuca, Loops of intervals and Darboux Baire 1 fixed point problem, ibid., to appear.

Department of Mathematics

Gdańsk University

Wita Stwosza 57

80-952 Gdańsk, Poland

E-mail: pszuca@radix.co.pl

Received 16 September 2002;

in revised form 21 August 2003 